\title{
C-09
}

\section{NORMATIVA DE TELECONTROL EN ZONAS REGABLES}

\author{
Madurga del Cura, Cristina $(P)^{1}$, Cervantes Díaz-Toledo, Alfonso ${ }^{2}$, Sánchez de Ribera \\ González, Alejandro ${ }^{3}$, Zazo Salinero, Teresa $^{4}$
}

\author{
${ }^{1}$ Ing. Sup. Agrónomo, CENTER, Camino de la Vega, s/n, 28830 San Fernando de Henares, \\ Madrid, cmadurga@tragsa.es \\ ${ }^{2}$ Ing. Tco. en Informática de Gestión, CENTER, Camino de la Vega, s/n, 28830 San \\ Fernando de Henares, Madrid, acervant@tragsa.es \\ ${ }^{3}$ Ing. Sup. Agrónomo, CENTER, Camino de la Vega, s/n, 28830 San Fernando de Henares, \\ Madrid, asanche4@tragsa.es \\ ${ }^{4}$ Ing. Sup. Agrónomo, CENTER, Camino de la Vega, s/n, 28830 San Fernando de Henares, \\ Madrid, tzazo@tragsa.es
}

\section{Resumen}

Para definir los requisitos mínimos de calidad y funcionalidad que deben tener todos los equipos de telecontrol que se instalan en campo es imprescindible que se genere una normativa en el ámbito de telecontrol. Por este motivo, desde el organismo de normalización AEN/CNT68/SC2 "Riegos" cuya Secretaría ostenta el MAGRAMA - CENTER, se está dando forma, junto con todos los implicados en el sector, a una norma que no excluya a nadie, pero que a su vez sirva para hacer una caracterización inequívoca de estos equipos, facilitando la elección del sistema más adecuado en función de las necesidades de cada regadío y asegurando su correcto funcionamiento. Actualmente se está trabajando tanto a nivel internacional (ISO) como a nivel nacional en la elaboración de dicha norma que contará con cuatro partes: Definiciones, Ensayos de funcionalidad y robustez, Interoperabilidad y Especificaciones.

\begin{abstract}
To define the minimum quality and functional requirements for all field remote control equipment to be installed in an irrigation system it's indispensable to have a Standard for Remote Monitoring and Control Systems. For this reason, the Normalization Organization AEN/CNT68/SC2 "Irrigation", the Secretariat of which is hold by MAGRAMA - CENTER, is preparing, in collaboration with all stakeholders in the business, a Standard which whilst not selective, it is also useful for a unique characterization of the equipment, making easier the selection of the most suitable system taking into account the needs of every irrigation system and guaranteeing its correct performance. Work is currently in progress at both an International level (ISO) and a National level to elaborate the Standard which will have four sections: Definitions, Functionality and Robustness Tests, Interoperability and Specifications.
\end{abstract}

\section{1- Introducción}

Uno de los objetivos fundamentales de la modernización de los regadíos, además de reducir el consumo de agua y mejorar la eficiencia en su aplicación, ha sido el mejorar la calidad de vida de los agricultores.

Ello ha supuesto la instalación de diferentes sistemas de telecontrol que permiten regar de forma automática y a cualquier hora del día, sin necesidad de actuar de forma 
manual sobre los equipos de riego y facilitando los trabajos de gestión y mantenimiento de los regadíos.

Sin embargo, la ausencia de normativa nacional/internacional que regule este tipo de sistemas, ha hecho que, en ocasiones, no haya sido fácil su puesta en marcha y que incluso, en algunos casos, se haya abandonado su uso.

Por este motivo, desde la Secretaría del Organismo de normalización AEN/CTN68/SC2 "Riegos", que desempeña el MAGRAMA - CENTER, se ha promovido la redacción de una norma nacional-europea-internacional, que establezca los requisitos mínimos que deben cumplir estos equipos en cuanto a funcionalidad e interoperabilidad.

Tanto a nivel nacional como internacional, se está trabajando en la redacción de la norma, que está previsto que tenga 4 partes.

En noviembre de 2007 se publicó la norma UNE-EN 15099-1 "Técnicas de riego. Telecontrol de zonas regables. Parte 1: "Consideraciones generales", vigente en Europa y España.

Actualmente se están elaborando las partes 2 y 3 de la norma, relativas a ensayos e interoperabilidad, y que son las que se describirán en esta ponencia.

La parte 2 de ensayos tiene como objetivo la evaluación de las unidades remotas, de modo que se pueda conocer su funcionalidad y operatividad con todo detalle, facilitando la elección de la más adecuada en función de las necesidades que tenga cada usuario y asegurando, de ese modo, unos requisitos mínimos de calidad.

En cuanto a la parte 3, este documento proporciona las directrices para implementar el modelo estandarizado aplicado al regadío, bajo cualquier plataforma tecnológica. De ese modo, se facilitará la comunicación entre los sistemas de control y gestión, independientemente del fabricante, aumentando las posibilidades de modificación, mejora o ampliación de las instalaciones, tanto existentes como futuras.

La última parte de la norma, relativa a especificaciones técnicas, se desarrollará en función del avance de las partes anteriormente citadas.

\section{2- Materiales y Métodos}

EI CENTER lleva a cabo una importante labor en materia de normalización de materiales y equipos de riego. Tal y como se ha dicho anteriormente, a nivel nacional, desempeña la Secretaría del AEN/CTN68/SC2 y, a nivel internacional, coordina diversos grupos de trabajo, entre los que cabe destacar el grupo de trabajo de telecontrol.

Esto ha permitido transmitir la necesidad de redactar una norma específica de telecontrol, norma en la que se está trabajando activamente en el CENTER, apoyándose de manera importante en el Laboratorio.

En los siguientes apartados se va a describir qué trabajos se han llevado a cabo para desarrollar la parte 2 y la parte 3 de este proyecto de norma.

\section{1- Parte 2: Funcionalidad y robustez} CENTER.

En el desarrollo de esta parte ha jugado un papel fundamental el Laboratorio del 
El Laboratorio Central para Ensayo de Materiales y Equipos de Riego, ubicado en el Centro Nacional de Tecnología de Regadíos (CENTER), órgano dependiente del Ministerio de Agricultura, Alimentación y Medio Ambiente (MAGRAMA), dispone de un laboratorio específico para sistemas de telecontrol, así como un banco de ensayo de electroválvulas que sirven de apoyo al desarrollo de estas normas.

Para la realización de los ensayos se dispone de un conjunto de equipos calibrados que permiten asegurar la exactitud y fiabilidad en los resultados.

De esta manera algunos de los equipos más representativos para la realización de las pruebas son:

- Osciloscopio: equipo para poder medir y representar gráficamente diferentes magnitudes escalares como puede ser la tensión. De esta manera, facilita algunas de las pruebas que se realizan como puede ser lectura de pulsos y carga del condensador en el accionamiento de una electroválvula, entre otras.

- Generador de pulsos: se emplea como verificación de las entradas digitales de los equipos. Permite generar pulsos con diferentes anchos, de manera síncrona y asíncronamente.

- Fuente de alimentación: se alimentan los equipos a diferentes tensiones para verificar hasta qué valores sigue operativa.

- Simuladores de 100 metros de cable: equipo que simula la impedancia de 100 metros de cable para asegurarse que el equipo de telecontrol es capaz de accionar electroválvulas y leer contadores a grandes distancias.

- Calibrador de lazo: Genera señales de 4 a $20 \mathrm{~mA}$ para que conectado a las entradas analógicas del terminal se garantice su correcta medida.

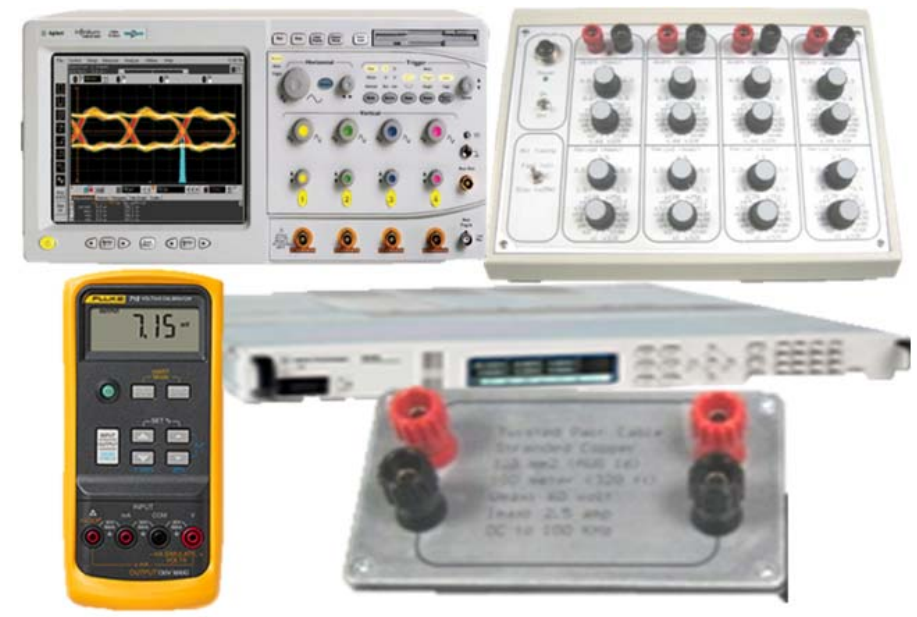

Figura 1: Osciloscopio, calibrador de lazo, generador de pulsos, fuente de alimentación y simulador de 100 metros de cable.

- Cámara climática: habitáculo donde se introducen los equipos remotos y someten a diferentes condiciones de temperatura y humedad. Así se verifica la correcta estanqueidad y funcionamiento de las unidades remotas con condiciones ambientales adversas.

- Banco de electroválvulas: circuito cerrado de agua con diferentes tipos de solenoides, de dos y tres hilos, y con diferentes voltajes de disparo. Cada 
electroválvula tiene un contador digital asociado. Con el equipo remoto conectado, se realizan aperturas y cierres verificando que el terminal remoto comande las órdenes y realice el conteo de los pulsos generados correctamente.

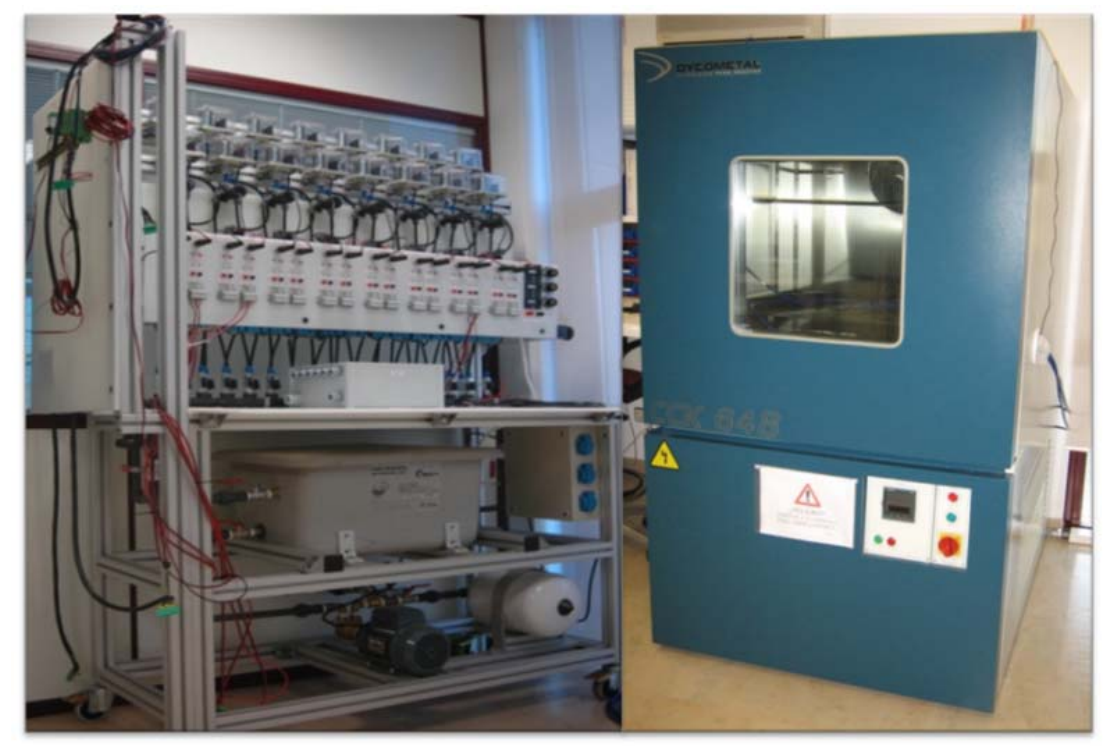

Figura 2: Banco de electroválvulas y cámara climática

\section{2- Parte 3: Interoperabilidad}

El desarrollo de esta parte de la norma se ha hecho, fundamentalmente, englobado dentro de un proyecto de I+D+i de la empresa TRAGSA, contando con la colaboración del personal del CENTER.

Aunque en este documento no se va a entrar en mucho detalle, puesto que se describe en la ponencia "Resultados prácticos de la aplicación de estándares industriales a la interoperabilidad en el regadío: Proyecto MEGA", sí que se van a definir los principales trabajos llevados a cabo hasta definir el primer borrador presentado en ISO en la pasada reunión celebrada en junio de 2015 en Madrid.

El objetivo fundamental, en este caso, es conseguir no depender de un único sistema de telecontrol sino que se permita la interoperabilidad entre diferentes sistemas, estandarizando el lenguaje y la arquitectura de los mismos.

Para alcanzar la interoperabilidad entre sistemas de control y de explotación de infraestructuras hidráulicas se definen los niveles y componentes identificados en la Figura 3. 


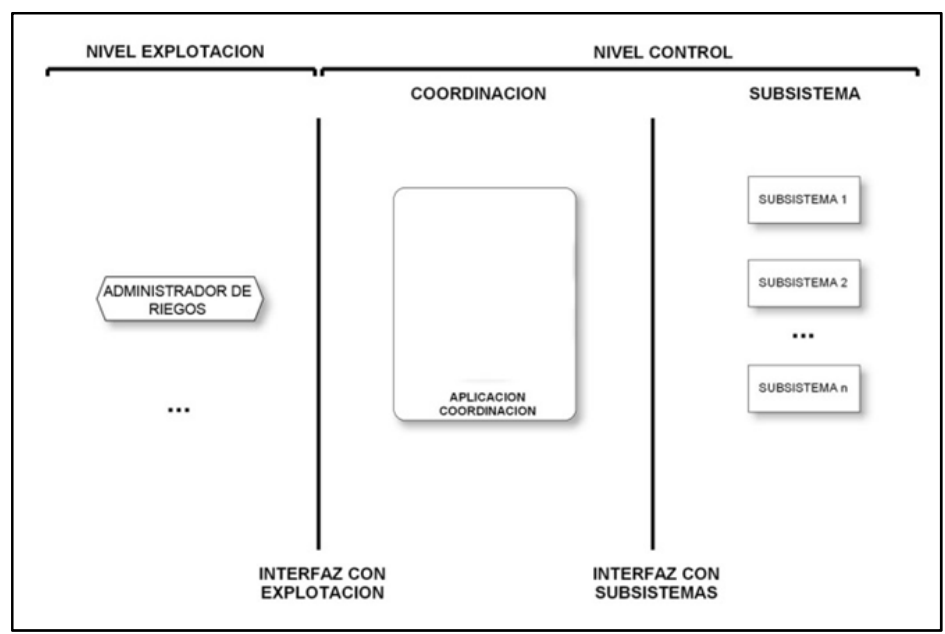

Figura 3: Niveles y componentes de una arquitectura interoperable sistemas.

Esto va a facilitar tanto el mantenimiento como el desarrollo continuo de estos

La nueva arquitectura pretende alcanzar una diferenciación clara dos partes de los sistemas muy diferentes, pero dependientes entre sí:

- Nivel de gestión/explotación, orientado a la toma de decisiones.

- Nivel de control, que incluye la transmisión y adquisición de datos, así como la ejecución de procesos.

Inicialmente se ha hecho un importante trabajo en la definición de las pruebas desde el punto de vista teórico y preparando todos los equipos necesarios para luego ejecutarlas en un banco de ensayo. El trabajo de programación es esencial en estas pruebas y ha llevado la mayor parte del tiempo, al igual que la adaptación de los equipos al sistema interoperable.

Las instalaciones de ensayo deben disponer de dos partes claramente diferenciadas pero ambas necesarias:

- Infraestructura informática para la verificación de interfaces. Las instalaciones deben disponer de testers para todos los interfaces definidos en el estándar. Esos testers emplearán los protocolos de comunicación definidos en los anexos descriptivos de los interfaces.

- Infraestructura hidráulica y sensórica para verificación de funcionalidades. Las instalaciones de ensayo deben disponer de todas las entidades hidráulicas modeladas en la norma. 


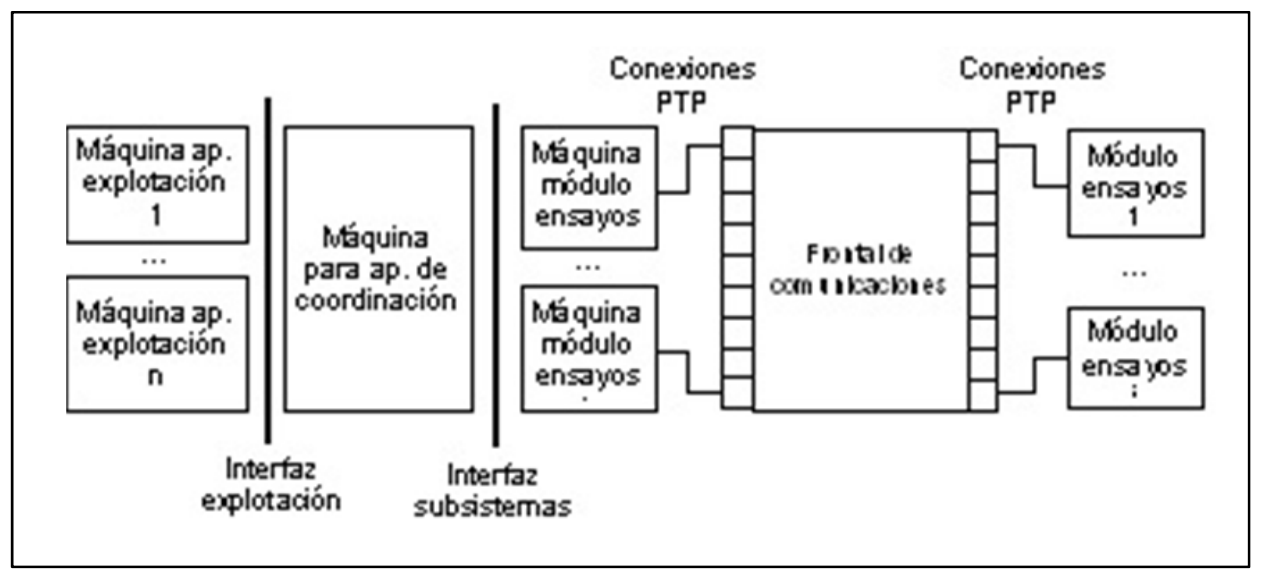

Figura 4: Componentes de la infraestructura de comunicaciones

En cuanto a la infraestructura hidráulica y sensórica, a día de hoy, sólo se ha implementado la unidad que se conoce como hidrante simple (HYS)

El módulo de pruebas del hidrante simple dispondrá de los siguientes dispositivos para verificar sus funcionalidades básicas:

- Totalizador de volumen;

- Elemento de corte.

Además, integrará otros dispositivos con dos finalidades diferenciadas:

- Dispositivos para comprobación de funcionalidades extendidas: transmisores de presión y otros sensores de parámetros climáticos (humedad relativa, temperatura, radiación solar, viento y pluviometría). El transmisor de presión convertirá la presión preferiblemente a una señal normalizada 4...20mA. Los sensores climáticos que puedan ser compatibles con un subsistema que controle una entidad tipo HYS, serán también de señal normalizada $4 \ldots 20 \mathrm{~mA}$. Estos elementos se conectarán a las entradas analógicas disponibles en el elemento de control.

- Dispositivos para la ratificación del correcto funcionamiento de la entidad hidráulica: detector de paso de agua (confirmación de seccionamiento abierto/cerrado) y detector de posición del elemento de corte (confirmación de seccionamiento abierto/cerrado). Ambos elementos serán llevados a una entrada digital del elemento de control.

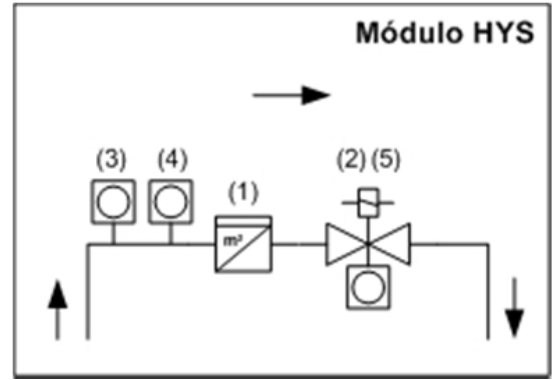

Figura 5: Diagrama de componentes del módulo HYS

Los componentes del módulo identificados en la Figura 5 son:

- (1) Totalizador de volumen 
- (2) Elemento de corte controlado por subsistema

- (3) Medidor de presión

- (4) Detector de paso de agua

- (5) Detector de posición de elemento de corte

La infraestructura hidráulica se ha implementado en Aula Dei, desarrollando un banco de ensayo que replica un sistema completo, desde la acometida hasta el hidrante, con el fin de poder extender la interoperabilidad a cualquier infraestructura típica del regadío.

En este año 2016 se han iniciado los ensayos en campo en el banco de pruebas de Aula Dei estando todavía en una primera fase de obtención de resultados.

En la figura 6 puede verse dicha instalación.

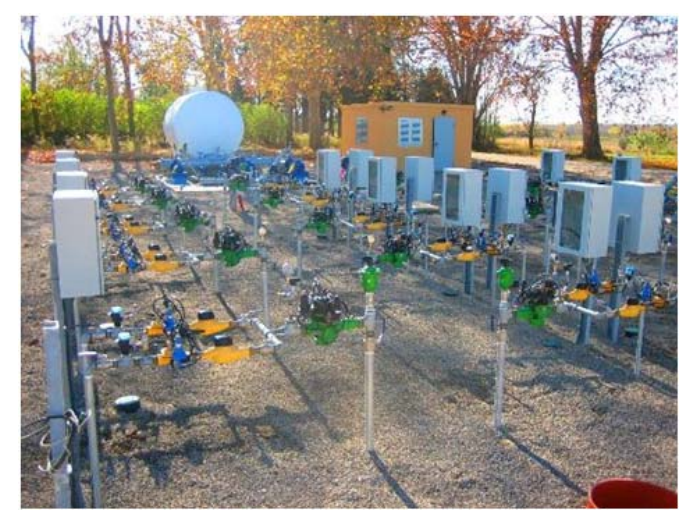

Figura 6: Banco de ensayo de Aula Dei

\section{3- Resultados y discusión}

Todo el trabajo y el esfuerzo dedicado en estas pruebas tenían como fin último la preparación de unos borradores que sirvieran como base a la norma de telecontrol.

Esta ardua labor no se ha concluido con la presentación en ISO de estos primeros documentos, sino que se sigue trabajando teniendo en cuenta los comentarios recibidos por parte de los expertos nacionales e internacionales, con el objetivo de publicar una norma útil para todo el sector.

A continuación se van a describir los ensayos que se reflejan en dichos documentos.

\section{1- Parte 2: Funcionalidad y robustez}

A lo largo de los últimos 10 años se han llevado a cabo numerosas pruebas a decenas de unidades remotas que han permitido ir definiendo los protocolos de ensayo para comprobar su funcionamiento, así como establecer los requisitos mínimos de calidad que deben exigirse.

Los ensayos se han dividido en funcionalidad, donde se verifica el correcto funcionamiento de los equipos, y robustez, donde se somete al terminal remoto a pruebas extremas para verificar los valores límite de las remotas. 
A modo de resumen, todos los ensayos se pueden dividir en seis grandes bloques:

1. Pruebas de alimentación:

Dentro de los ensayos de alimentación destacan tres:

- Alimentación a diferentes tensiones: Con estas pruebas se comprueba qué sucede con las remotas cuando están sometidas a diferentes tensiones de alimentación.

- Pérdidas de alimentación: La pérdida brusca de alimentación de la remota no debe suponer una pérdida de valores críticos, como son los programas de riego y los valores de contadores. Restableciendo la alimentación la remota debe seguir funcionando con normalidad, continuando la ejecución de los programas de riego que tuviera.

Se programa un riego y se procede a interrumpir la alimentación en los instantes reflejados en la Figura 7, comprobando posteriormente la respuesta de la remota.

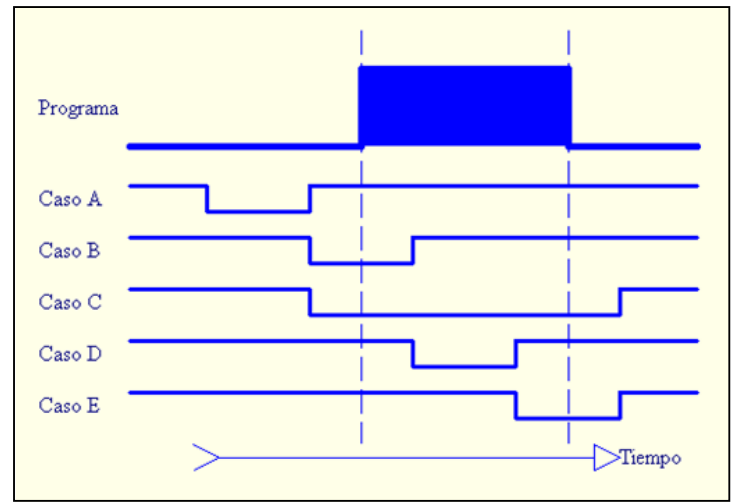

Figura 7: Diferentes casos de pérdida de alimentación para el ensayo de programaciones

2. Inversión de polaridad:

Si la remota soporta una inversión accidental de la polaridad, se invierte la polaridad en los cables que conectan la fuente de energía con la remota. Se mantiene durante 1 minuto y se comprueba que, al restablecerse la alimentación nominal, la remota sigue operativa.

\section{Consumos.}

Las pruebas de consumos suponen un punto importante en telecontrol. La razón fundamental es que, al ser equipos instalados en campo y no disponer de abastecimiento eléctrico, se requiere el uso de baterías recargables o de pilas. Para que la duración en tiempo de vida de los equipos sea el máximo posible, es necesario que los terminales remotos tengan unos consumos adecuados para poder alargar la vida útil de las baterías. Por este motivo es importante verificar los consumos que se producen cuando se realizan actuaciones y cierres de electroválvulas, lecturas de contador, lecturas de entradas analógicas y más importante si cabe, cuando se producen las comunicaciones.

4. Estudio de las condiciones de trabajo. 
La remota debe estar preparada para trabajar a la intemperie, en una arqueta en el campo, en condiciones extremas de humedad y temperatura. Esto exige que tenga, al menos, un grado de protección IP65 una vez conectados cables de entradas y salidas en las conexiones de la remota. Además, debe ser capaz de llevar a cabo todas sus funciones, en todo el rango de temperaturas especificado por el fabricante.

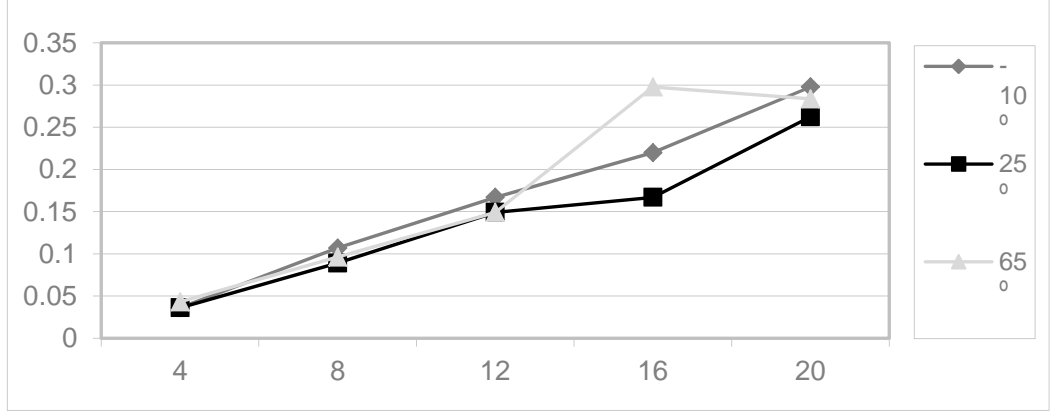

Figura 8: Desviación de la entrada analógica con respecto a la temperatura

Se verificará la capacidad de la remota de accionar electroválvulas, y la fiabilidad en la lectura de contadores y la estabilidad en las medidas de las entradas analógicas.

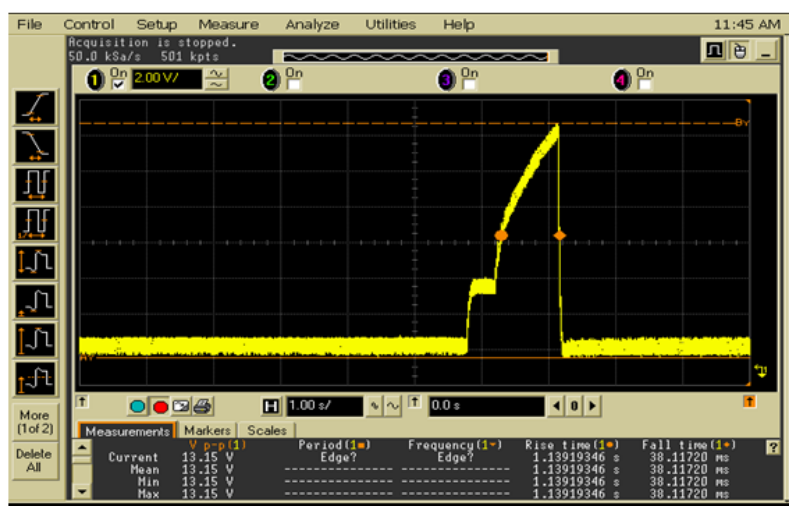

Figura 9: Gráfica del proceso de disparo de una salida digital de la unidad remota

5. Protección ante situaciones anómalas.

Supone un punto importante conocer al detalle el funcionamiento de los equipos remotos. Hay que tener en cuenta qué sucede si se producen, por ejemplo en el momento de la instalación de los equipos, errores accidentales de cableado, como el intercambio de los cables entre las entradas y salidas.

Es importante, además, conocer qué sucede si se cortocircuitan las salidas digitales o las entradas analógicas.

Para que el resultado de la prueba sea positivo es necesario que la remota, una vez realizados los cortocircuitos en las entradas/salidas, siga funcionando correctamente sin verse afectada.

El módem/radio supone otro factor de riesgo en los terminales remotos. Es importante que los equipos no se vean afectados si se produce un cortocircuito o si se queda en circuito abierto.

6. Comportamiento a largo plazo 
Con esta prueba se pretende probar la fiabilidad de la remota tras un uso intensivo y en los límites máximos admisibles especificados por el fabricante.

Las pruebas de fiabilidad se realizan a temperatura ambiente con una remota, alimentada desde una fuente de alimentación regulable y utilizando el banco de pruebas de electroválvulas/solenoides. La prueba realiza 18.250 ciclos de aperturas y cierres de solenoides, pensando en un tiempo de vida del equipo de 50 años, realizando 2 actuaciones diarias.

Posteriormente, se procede a leer el valor del contador almacenado por la remota y se verifica que son los mismos que aparecen en el contador digital del banco de electroválvulas. Se espera que se produzcan todos los disparos comandados a los solenoides y la lectura de pulsos de contador, con un error inferior al 0,05\%, para comprobar la fiabilidad del sistema.

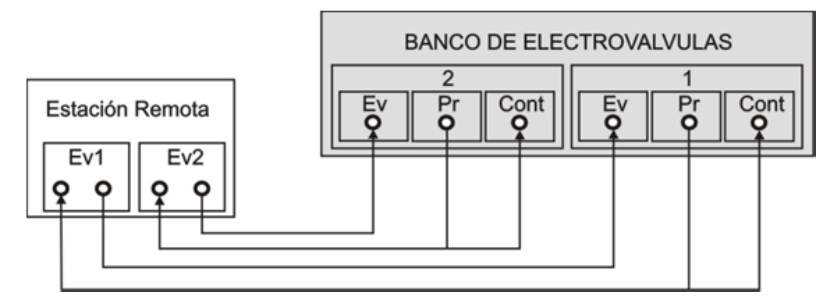

Figura 10: Conexionado de Estación Remota a Banco de EV para Prueba de comportamiento a largo plazo.

\section{2- Parte 3: Interoperabilidad}

Los ensayos comprenden un conjunto de pruebas informáticas e hidráulicas a las que se someten los subsistemas, las aplicaciones de coordinación y las aplicaciones de explotación. Dichos ensayos se dividen en dos fases:

- Fase 1. Pruebas informáticas de verificación de interfaz. Se verificará que los mensajes (tanto peticiones como respuestas) que intercambia el elemento objeto de los ensayos con el resto de componentes de la arquitectura respetan el estándar.

- Fase 2. Ensayos de campo para verificación de funcionalidades. Una vez superada la fase anterior, se verificará que el elemento objeto de los ensayos ejecuta correctamente aquellas funcionalidades asociadas a la entidad hidráulica que éste puede explotar o controlar.

Se entenderá como adaptado al estándar aquel producto que supere ambas fases, verificándose que es capaz de realizar todas las funciones exigibles para el control o explotación de la/s entidad/es hidráulica/s definidas en el estándar.

El primer borrador de los ensayos se presentará en la reunión del ISO TC23/SC18 que se celebrará a mediados de mayo en Montpellier.

\section{4- Conclusiones}

Las normas tienen como uno de sus objetivos fundamentales garantizar la calidad de los productos y sistemas. Por ello las normas deben definir una serie de criterios técnicos que van a servir para caracterizar un producto y establecer sus requisitos mínimos de 
calidad. De esa manera, se facilita también la comercialización y la certificación, si fuese necesario.

Eso es lo que se ha pretendido a la hora de redactar la norma de telecontrol, que los equipos que forman parte de estos sistemas, estén regulados de alguna forma y, así, poder asegurar un correcto funcionamiento en las redes de riego.

Hay que destacar, además, el gran interés que ha despertado este tema a nivel internacional, ya que países tan importantes en el regadío como Israel, USA o India que siempre han ido un paso por detrás de España en materia de telecontrol, están trabajando muy intensamente en el desarrollo de estos sistemas con el objetivo de aplicar las más avanzadas tecnologías al regadío.

Como se ha comentado, este es un trabajo que está muy vivo y es importante contar con la participación de todos los implicados en el sector, de forma que se consiga una norma útil para todos. 